\title{
Endodontic Management of Maxillary First Molar with 7 Canals - A Case Report
}

\author{
Ajit Hindlekar ${ }^{1}$, Srinidhi SR ${ }^{2 *}$, Jay Khatri ${ }^{3}$, Niranjan Desai ${ }^{4}$ and Omkar Balsaraf \\ ${ }^{1}$ Dept of Conservative Dentistry \& Endodontics, Sinhgad Dental College \& Hospital, India \\ ${ }^{2}$ Professor \& Head, Dept of Conservative Dentistry \& Endodontics, Sinhgad Dental College \& Hospital, India \\ ${ }^{3}$ Wonder Smile Dental Clinic, Pune, India \\ ${ }^{4}$ Dept of Conservative Dentistry \& Endodontics, Sinhgad Dental College \& Hospital, India \\ ${ }^{5}$ Lecturer, Dept of Conservative Dentistry \& Endodontics, Sinhgad Dental College \& Hospital, India
}

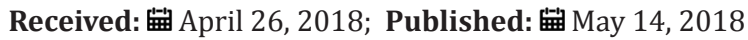

*Corresponding author: Srinidhi SR MDS, Professor and Head, Dept of Conservative Dentistry \& Endodontics, Sinhgad Dental College \& Hospital, Pune, Maharashtra, India

\begin{abstract}
Comprehensive knowledge about the normal anatomy of the root canal system and its variations of the teeth are essential for the clinician as aberrations in number, shape and size are very common. Various diagnostic aids like preoperative radiographs in multiple angulations or the Cone Beam Computed Tomography can help the clinician in location and effective debridement of the root canal system. This case report describes a rare occurrence of 7 canals in a maxillary first molar in which CBCT was used as an additional diagnostic tool.
\end{abstract}

\section{Introduction}

Knowledge of canal morphology and its frequent variations is a prerequisite for endodontic therapy. These morphological variations in root canal anatomy play an important role in the outcome of root canal therapy. The foremost common causes of treatment failures, especially in permanent maxillary first molars have been attributed to the inability to locate, clean and adequately fill all canals of the root canal system [1]. The objective of root canal treatment is thorough cleaning and shaping of all the pulp spaces to be filled with an inert filling material. A successful root canal treatment depends on the diagnosis, treatment planning, good knowledge of the root canal system, and its frequent variations [2].

There are case reports of the incidence of second mesiobuccal (MB2) canals ranging between 18 and 96.1\% [3-5]. The incidence of five canals is reported to be $2.25-2.4 \%[6,7]$ and the incidence of six canals is reported to be $0.32-0.88 \%$ [8]. The incidence of a second distobuccal (DBI) canal in the maxillary first molars has been reported to be as low as $1.7 \%$ [9] to $1.25 \%$ [10]. Case reports with more than four root canals have also been reported in threerooted maxillary first molars. An ex-vivo study by Baratto Filho, reported one maxillary first molar out of 140 samples having three roots and seven canals. They identified three mesiobuccal canals, three distobuccal canals, and one palatal canal [11]. Kottoor et al reported the endodontic management of the maxillary first molars with seven and eight canals, respectively $[12,13]$. This case report discusses the endodontic management of an unusual root canal configuration in a maxillary first molar showing three roots and seven canals. This unusual morphology was confirmed with the help of cone beam computerized tomography (CBCT) scans.

\section{Case reports}

A 26-year-old male patient reported to the Department of Conservative Dentistry and Endodontics, with the chief complaint of spontaneous pain in the upper right posterior region of the jaw for the past five days. The patient gave a history of intermittent pain for the last two months, which had increased in intensity in the 
preceding five days. The tooth gave prolonged sensitivity to hot and cold. Clinical examination showed a deep occlusal carious lesion, tenderness on percussion with no mobility. Sensitivity testing of the involved tooth with heated gutta-percha (Dentsply Maillefer, Ballaigues, Switzerland) caused an intense lingering pain, whereas, an electronic pulp testing (Parkell, USA) gave an early response. A preoperative radiograph (Figure 1) revealed occlusal radiolucency approaching the pulp space, with the periodontal ligament space widening in relation to the mesiobuccal root. From the clinical and radiographic examination, a diagnosis of symptomatic irreversible pulpitis with symptomatic apical periodontitis was made and nonsurgical endodontic treatment was recommended. Radiographic evaluation of the involved tooth with multiple angulations did not indicate any variation in the root canal anatomy. Local anesthesia was induced using $1.8 \mathrm{ml}$ of $2 \%$ Lignocaine and Adrenaline (Aqua Fine Injecta Pvt. Ltd., Pune, India). A rubber dam was placed and a conventional endodontic access opening established.

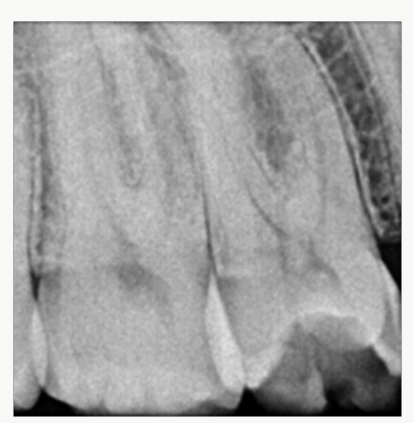

Figure 1: Preoperative radiographic View.

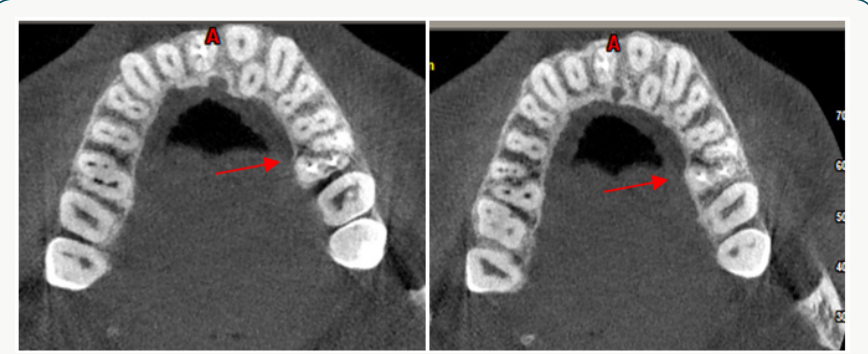

Figure 2a\&2b: Coronal Section \& Apical Section.

CBCT view

Initially, the MB1, MB2, DB1 and Palatal canals (mesio palatal) were located. Examination of the floor of the pulp chamber under a dental operating microscope (DOM) (Moller Wedel, Germany) showed three additional root canal orifices (MB3, DB2, and Disto palatal). Conventional triangular access was modified to a trapezoidal shape to improve access to the additional canals. The patency of the canal was confirmed with ISO \#10 K-files (Mani, Japan). To confirm this unusual morphology, it was decided to perform CBCT imaging of the tooth. A sterile cotton pellet was placed in the pulp chamber and Cavit G (3M ESPE Dental Products, St Paul, MN) was used to seal the access cavity. Informed consent was obtained from the patient, and a multislice CBCT scan of the maxilla was performed with a tube voltage of $100 \mathrm{KV}$ and a tube current of $8 \mathrm{~mA}$. The involved tooth was focused and the morphology was obtained in the transverse, axial, and sagittal sections, of $0.5 \mathrm{~mm}$ thickness. The CBCT scan slices revealed seven canals (three mesiobuccal, two palatal, two distobuccal) (Figures 2a \& 2b).

At the next appointment, the working lengths were determined with the help of an apex locator (Root ZX, J Morita, Tokyo, Japan), under rubber dam isolation. Intraoral periapical radiographs of the mesiobuccal, distobuccal, and palatal root were taken to confirm the working lengths (Figure 3a). Cleaning and shaping was performed using nickel-titanium rotary instruments (M2, Dentsply VDW, Germany) using the crown-down technique. During root canal preparation, irrigation was performed using $2.5 \%$ sodium hypochlorite solution. Final rinsing of the canals was performed using 2\% chlorhexidine digluconate (Asep RC, Stedman Anabond, Chennai, India) together with passive ultrasonic agitation. A saline wash was performed. The canals were dried with absorbent points (Dentsply Maillefer, Ballaigues, Switzerland), master cone fit verified (Figure 3b). Obturation was performed using cold lateral compaction and AH Plus resin sealer (Dentsply deTrey, Konstanz, Germany) (Figure 4). A radiograph was taken to establish the quality of the obturation. After completion of root canal treatment, the tooth was restored with a posterior composite filling (Matrix, Medicept Dental Products, UK) and the patient was advised to have a full coverage porcelain crown. The patient was asymptomatic at the two-month follow-up.

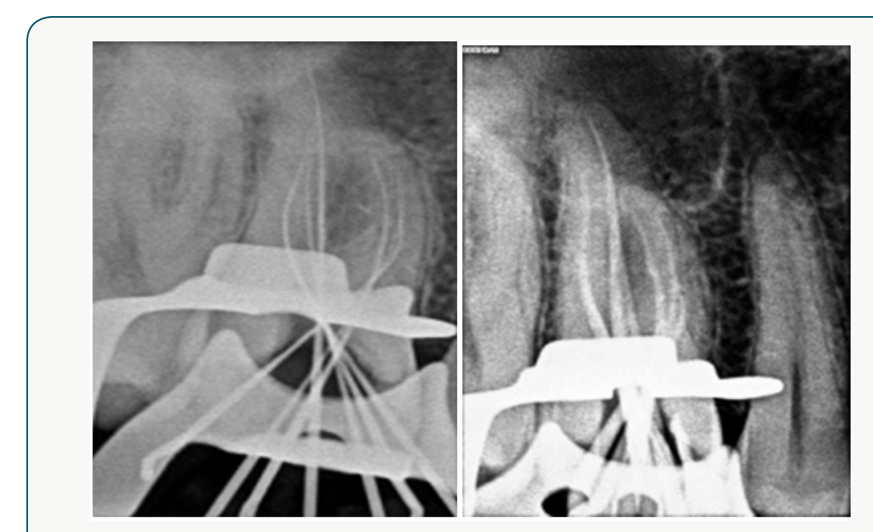

Figure 3a\&3b: Working length radiograph \& Master cone radiograph.

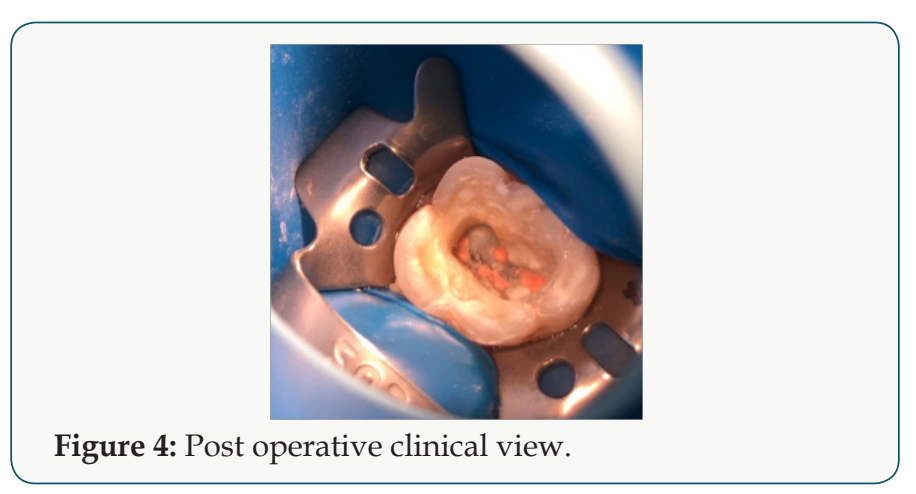




\section{Discussion}

Vertucci identified eight pulp space configurations in permanent teeth [14]. Additionally, 14 new additional canal types were reported by Sert and Bayirli, highlighting the complexity of the root canal systems [15]. In this case, CBCT scanning was used for a better understanding of the complex root canal anatomy and helped to confirm the presence of multiple canals. The CBCT axial images showed the mesiobuccal root had a Sert and Bayirli type XVI canal configuration [15]. The distobuccal root presented with a Vertucci type II canal pattern [14]. The palatal root showed a Vertucci IV canal pattern [14]. In the apical area, the MB2 and MB3 joined together to exit as one and the DB1 and DB2 joined to exit as one. In clinical conditions, conventional periapical radiographs and digital radiographs taken in different angulations are an essential part of endodontic treatment for the identification of root and canal configuration. Advanced techniques like computed axial tomography scanning are being used increasingly to evaluate root canal morphology as a three-dimensional image. It allows the operator to view the roots and their canal systems at different levels [16], which helps to identify a larger number of morphological variations than in the conventional radiographs [17]. In the present case, the use of a dental operating microscope helped in the location and identification of additional canal orifices. As an additional finding, the contralateral maxillary first molar had additional root canals, which were clearly appreciable in the CBCT.

\section{Conclusion}

The root anatomy is highly complex and unpredictable. Clinicians should be constantly on the lookout for variations in anatomy, as the successful outcome of any case depends on the complete debridement and disinfection of all the canals.

\section{References}

1. (1969) Root of the maxillary first molar and its endodontic significance. Oral Surg Oral Med Oral Pathol 28: 419-425.

2. Raghavendra SS, Hindlekar AN, Desai NN, Vyavahare NK, Napte BD (2014) Endodontic management of maxillary first molar with seven root canals diagnosed using Cone Beam Computed Tomography scanning. Indian J Dent 5(3): 152-156.

3. Kulild JC, Peters DD (1990) Incidence and configuration of canal systems in the mesiobuccal root of maxillary first and second molars. J Endod 16(7): 311-317.
4. Buhrley LJ, Barrows MJ, Be Gole EA, Wenckus CS (2002) Effect of magnification on locating the MB2 canal in maxillary molars. J Endod 28(4): 324-327.

5. Walton R, Torabinejad M (2002) In: Principles and practice of Endodontics. $3^{\text {rd }}(E d n)$. Philadelphia: WB Saunders Co p. 181-233.

6. Gray R The maxillary first molar. In: Bjorndal A, Skidmore E (Eds). Anatomy and Morphology of the Human Teeth. Iowa City: University of Iowa College of Dentistry p. 31-40.

7. Vigouroux ASA, Bosaans TSA (1978) Anatomy of the pulp chamber floor of the permanent maxillary first molar. J Endod 4(7): 214-219.

8. Martinez-Berna A, Ruiz-Badanelli P (1983) Maxillary first molars with six canals. J Endod 9(9): 375-381.

9. Cleghorn BM, Christie WH, Dong CC (2006) Root and root canal morphology of the human permanent maxillary first molar: A literature review. J Endod 32(9): 813-821.

10. Kim Y, Lee SJ, Woo J (2012) Morphology of maxillary first and second molars analyzed by cone-beam computed tomography in a Korean population: Variations in the number of roots and canals and the incidence of fusion. J Endod 38(8): 1063-1068.

11. Baratto Filho F, Zaitter S, Haragushiku GA, De Campos EA, Abuabara A, et.al (2009) Analysis of the internal anatomy of maxillary first molars by using different methods. J Endod 35(3): 337-342.

12. Kottoor J, Velmurugan N, Sudha R, Hemamalathi S (2010) Maxillary first molar with seven root canals diagnosed with cone-beam computed tomography scanning: A case report. J Endod 36: 915-921.

13. Kottoor J, Velmurugan N, Surendran S (2011) Endodontic management of a maxillary first molar with eight root canal systems evaluated using cone-beam computed tomography scanning: A case report. J Endod 37(5): 715-719.

14. Vertucci FJ (2005) Root canal morphology and its relationship to endodontic procedures. Endod Topics 10: 3-29.

15. Sert S, Bayirli GS (2004) Evaluation of root canal configurations of the mandibular and maxillary permanent teeth by gender in the Turkish population. J Endod 30(6): 391-398.

16. Patel S (2009) New dimensions in endodontic imaging: Part 2. Cone beam computed Tomography. Int Endod J 42(6): 463-475.

17. Robinson S, Czerny C, Gahleitner A, Bernhart T, Kainberger FM (2002) Dental CT evaluation of mandibular first premolar root configurations and canal variations. Oral Surg Oral Med Oral Pathol Oral Radiol Endod 93(3): 328-332. 
This work is licensed under Creative Commons Attribution 4.0 License

To Submit Your Article Click Here:

Submit Article

DOI: 10.32474/MADOHC.2018.02.000136

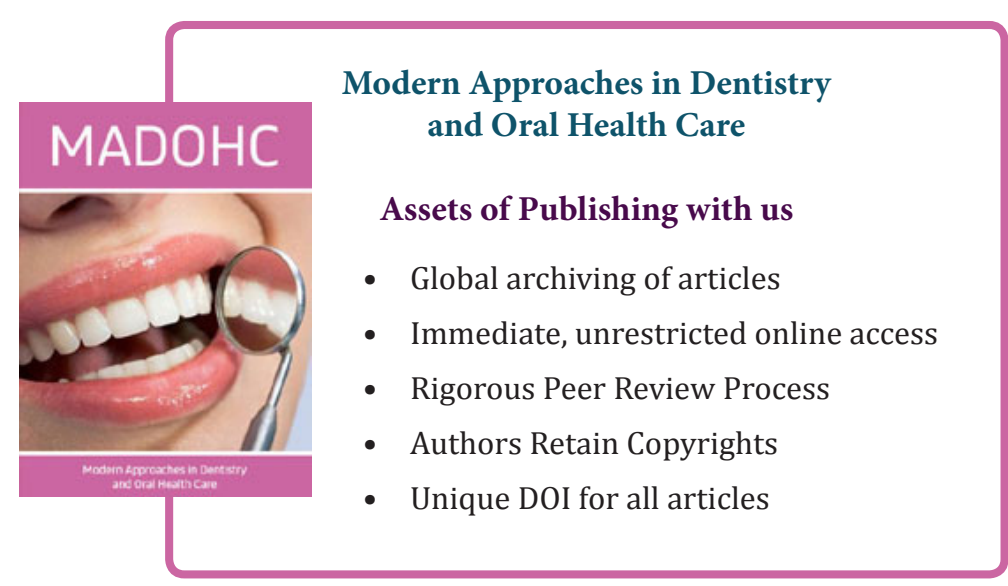

